\title{
A Practical Radar Climograph SimulationAlgorithm
}

\author{
LI Shu-hua \\ QingDao Branch of NAEI, QingDao 266041,China
}

\begin{abstract}
This paper puts forward an algorithm for simulating radar climograph which applys multi-typeinterpolation curvesto construct the contour of theradar climograph. The experimental resultsshow that the method is simple and can create good climograph.
\end{abstract}

Keywords: Meteorologic radar;Climograph simulation; multi-type interpolation

\section{Introduction}

In weatherRadar simulation system, we need to creat the climograph. This is different from the natural cloud because the climographmust identify the different partsof the cloud related to the rainwater intension in addition to simulate the shape and the variety of the cloud.

Most existing algorithms work for natural cloud simulation. We can list several representative ones: Blinn put forward a light reflection function for simulation of clouds and dusty surface[1];Maxsimulatednatural phenomena by gravity field[2];Kajiya etal designed a ray tracing algorithm to trace objects represented by densities within a volume grid, e.g. clouds, fog, flames, dust, particle systems[3]; Qi yue etal presented a method of rendering clouds with perlin Noise[4];Ruan Kun etal. delivered a cloud model based on midpoint deflection [5]; a Diamond-Square algorithm was brought forward by Shi Jian-di etal [6]. However, These methods are not fit for meteorologic radar simulation.

In this paper, we try to resolve the radar climograph simulation problem.Our algorithm is described in section II and several of the experimental results are given in section III before concluding in Section IV.

\section{The algorithm}

The basic idea of our method is to set out random points at first and then apply multi-type interpolation to rebuilt the contour of cloud. After having drawn the outline of the three layers of the radar climograph, each layer is filled in different color to indicate the rainwater intension in the cloud according to system defined rules. The algorithm consists of thefollowing steps:

1. Generate randompoints as a sequence;

2.Execute the multi-type interpolationto rebuilt the contour of the climograph; 
3.Creat multi-layers and fill different color in different layer to indicate the rainwater intension in the cloud.

Firstly randompoints with predefined inside size is generated. In order to avoid the intersection of the connecting lines, we select the points by circulatingaround the cloud centerat a random angle and the radius is also chosen randomly within the predefined length.

To simulate the climograph vividly, we apply multi-type interpolation curves to generate the cloud contours. These interpolation curves includethe spline curves, the fractualcurves[7], the Hermite curves and straight lines which is chosen randomly.

After the primary contour is generated, the Random points and multi-type interpolation procedure are repeated twice more to form the inboard contours. It is different from the periphery contour generation in its judging whether the selected point is within the neighbour periphery contour. If the point is an outliner, it will be aborted and a new one will be generated randomly.

The last step is to fill different color in different layer, that is, to color in the three areas segmented by the three contours.

\section{Experiments}

In this section experiments of our algorithm is executed using the Matlab software. Fig.1 shows some of the simulated climographs. In these experiments,yellow, purple and red are used sequently to identify the area where the rainwater intensity is more and more intense according to the system requirements. 


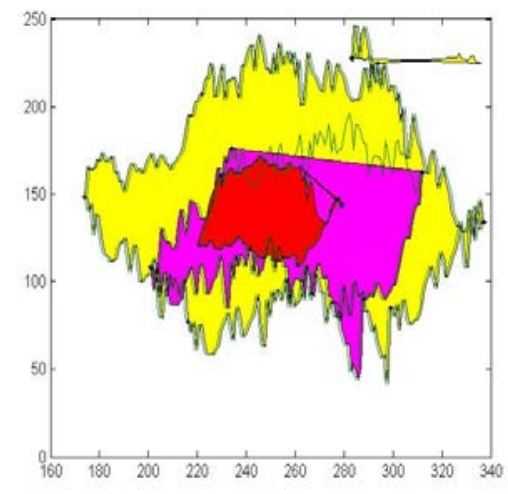

(a)

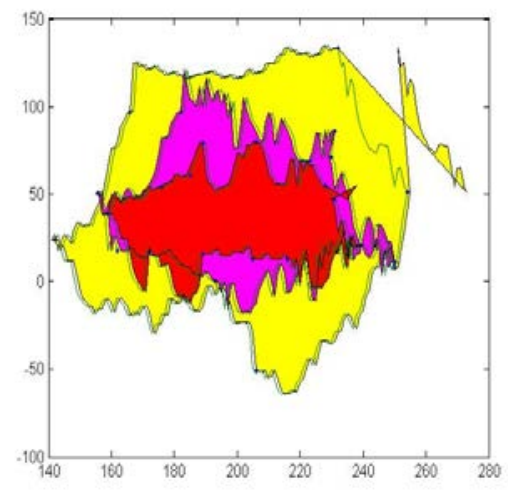

(b)

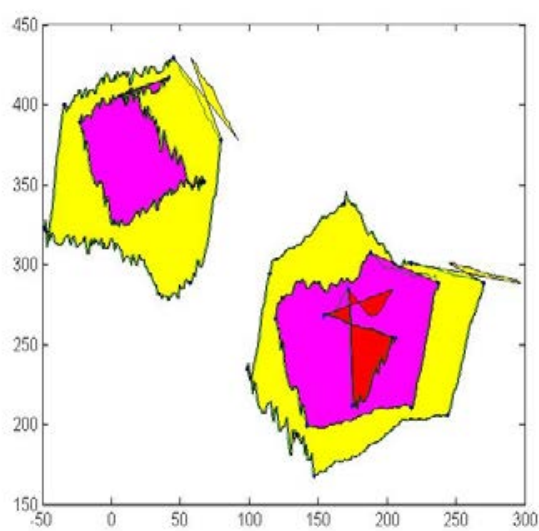

(c)

Fig.1 some of the simulated climographs

In Fig.1, the selected pointspointes are marked by blue dots.Fig.2 demonstrates some of the multi-type interpolation generated curves. 


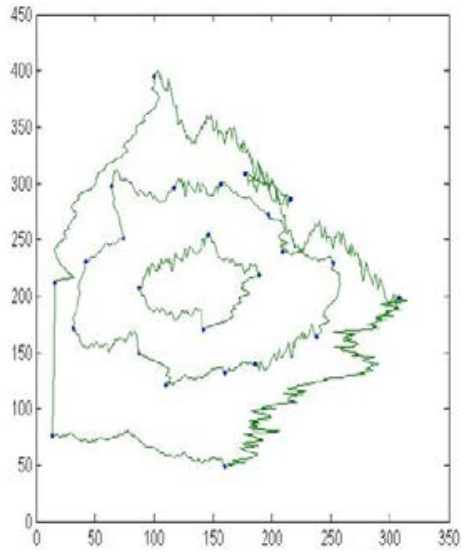

(a)

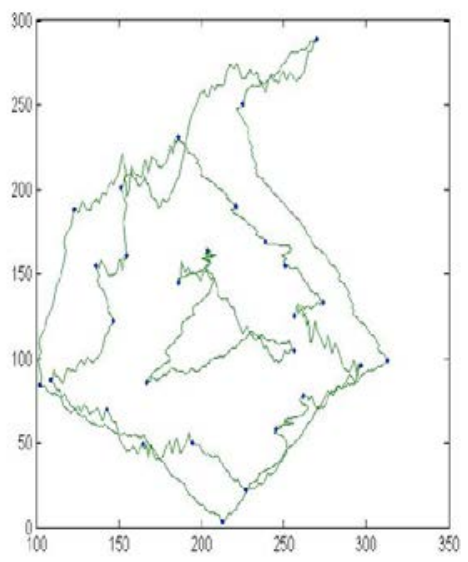

(c)

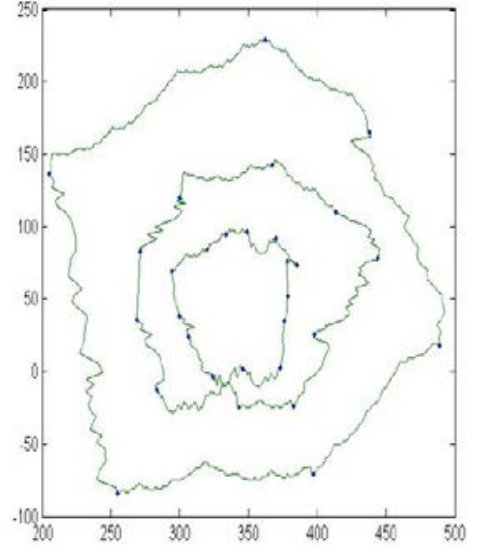

(b)

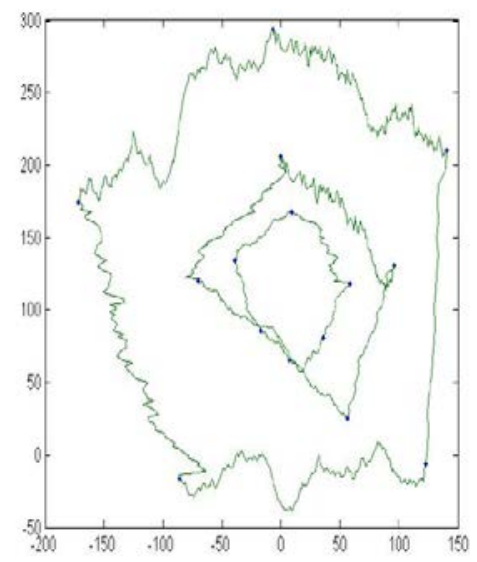

(d)

Fig.2multi-type interpolation generated contours

\section{Conclusion}

In this paper, we demonstrate a method to implement theclimograph simulation.Our algorithm is good at simulating the shape and the uniform diffusion property of the cloud, but it fails to creat cloud features in very bad weather. We will improve this in furture work. 


\section{References}

[1] J F Blinn. Light Reflection Function for Simulation of Clouds and Dusty Surface. Computer Graphics, 1982, 16 (3) : 21 - 29.

[2] N Max. The Simulation ofNatural Phenomena Panel. Computer Graphics, 1983, 17 (3) : 137 - 139.

[3] J T Kajiya, H B P Von. Ray Tracing Volume Densties. Computer Graphics, 1984, 18 (3) : 165 - 173.

[4] QI Yue, SHEN Xu-kun, DUAN Mi-yi, CHENG Hui-lin.A Method of Rendering Clouds with Perlin Noise. JOURNAL OF SYSTEM SIMULATION, 2002, 14 (9) : 1204 - 1207.

[5] Ruan Kun,Fan Yin. Modeling Cloud Based on Midpoint Deflection Algorithm. JOURNAL OF PLAUNIVERSITY OF SCIENCE AND TECHNOLOGY(NATURAL SCIENCE EDITION), 2003, 4 (1) : 99 - 102.

[6] Shi Jian di, Jiang Yi ming. Simulation of Dynamic Cloud Based on Multi-type Geometry. COMPUTER SIMULATION , 2006, vol 23(4):197-200.

[7] Fisher, Yuval, Multi-type Image Compression: Theory and Application, Springer-Verlag, New York, 1995. 